\title{
Achievement of recommended glucose and blood pressure targets in patients with type 2 diabetes and hypertension in clinical practice - study rationale and protocol of DIALOGUE
}

\author{
Anselm K Gitt ${ }^{1,2^{*}}$, Roland E Schmieder ${ }^{3}$, Eva Duetting ${ }^{4}$, Peter Bramlage ${ }^{5}$, Steffen Schneider ${ }^{1}$ and \\ Diethelm Tschöpe ${ }^{6,7}$ DIALOGUE Study Group*
}

\begin{abstract}
Background: Patients with type 2 diabetes have 2-4 times greater risk for cardiovascular morbidity and mortality than those without, and this is even further aggravated if they also suffer from hypertension. Unfortunately, less than one third of hypertensive diabetic patients meet blood pressure targets, and more than half fail to achieve target $\mathrm{HbA} 1 \mathrm{c}$ values. Thus, appropriate blood pressure and glucose control are of utmost importance. Since treatment sometimes fails in clinical practice while clinical trials generally suggest good efficacy, data from daily clinical practice, especially with regard to the use of newly developed anti-diabetic and anti-hypertensive compounds in unselected patient populations, are essential. The DIALOGUE registry aims to close this important gap by evaluating different treatment approaches in hypertensive type 2 diabetic patients with respect to their effectiveness and tolerability and their impact on outcomes. In addition, DIALOGUE is the first registry to determine treatment success based on the new individualized treatment targets recommended by the ADA and the EASD.

Methods: DIALOGUE is a prospective observational German multicentre registry and will enrol 10,000 patients with both diabetes and hypertension in up to 700 sites. After a baseline visit, further documentations are scheduled at 6 , 12 and 24 months. There are two co-primary objectives referring to the most recent guidelines for the treatment of diabetes and hypertension: 1) individual HbA1c goal achievement with respect to anti-diabetic pharmacotherapy and 2) individual blood pressure goal achievement with different antihypertensive treatments. Among the secondary objectives the rate of major cardio-vascular and cerebro-vascular events (MACCE) and the rate of hospitalizations are the most important.
\end{abstract}

Conclusion: The registry will be able to gain insights into the reasons for the obvious gap between the demonstrated efficacy and safety of anti-diabetic and anti-hypertensive drugs in clinical trials and their real world balance of effectiveness and safety.

Keywords: Type-2 diabetes, Hypertension, Efficacy, Effectiveness, Safety, Vildagliptin

\footnotetext{
* Correspondence: gitta@klilu.de

${ }^{1}$ Stiftung Institut für Herzinfarktforschung Ludwigshafen, Bremser Strasse 79, 67063, Ludwigshafen, Germany

${ }^{2}$ Herzzentrum Ludwigshafen, Med. Klinik B, Ludwigshafen, Germany

Full list of author information is available at the end of the article
} 


\section{Background}

There is an increasing prevalence of type-2 diabetes, which is attributable to a growing population, an increase in life expectancy, increased diagnostic efforts, and a reduced diabetes attributable risk due to recent advances in diabetes treatment [1]. Hypertension is the most frequent (90\%) among the co-morbid disease conditions [2] and further increases the risk for disease and treatment related complications [3]. The combination of both hypertension and diabetes accelerates the progression of diabetes related complications such as diabetic nephropathy, retinopathy, left ventricular hypertrophy, and diastolic heart failure and doubles the risk of stroke, $\mathrm{CV}$ and all-cause mortality as compared to non-diabetic patients with hypertension. Risk prediction in this population is however not easy making the adaption of available risk prediction tools necessary, partly because risks have gone down in recent years [4].

\section{Glucose and blood pressure control}

A number of studies have shown improved glycaemic control to be able to delay the onset and also to halt the progression of micro-vascular complications such as diabetic retinopathy and nephropathy. This has also been shown for neuropathic secondary disorders $[5,6]$. For macro-vascular complications, however, the picture to date is less straightforward. There is doubt as to whether the benefit of tight glycaemic control on macro-vascular events is as great as the one effected by blood pressure and lipid control. A recent meta-analysis of five large trials [7] found a significant reduction in event rates for non-fatal myocardial infarction and also for CAD, but all-cause mortality and stroke rates were not similarly affected.

An exploratory analysis of the United Kingdom Prospective Diabetes Study (UKPDS) data showed that the risk of both micro- and macro-vascular complications of type 2 diabetes (T2D) was strongly associated with the mean systolic blood pressure and that blood pressure lowering by $10 \mathrm{mmHg}$ led to a $15 \%$ reduction in the risk for death related to diabetes [8].

In the Steno-2 study, intensified therapy of the modifiable risk factors in patients with T2D and microalbuminuria was compared to standard treatment. In addition to lifestyle changes and diet modifications, all patients in this group received ACE-inhibitors (ACEI) or angiotensin receptor blockers (ARB) irrespective of baseline BP values and a vitamin-mineral supplement. The target limits for HbA1c, fasting cholesterol and triglycerides and blood pressure were much stricter than in the control group. This multi-factorial approach led to significant reductions in both micro- and macro-vascular event rates $[9,10]$.
Thus, in diabetic patients with hypertension, appropriate blood pressure control as well as glucose control is important. Current guidelines [11,12] recommend a multi-factorial approach with simultaneous targeting of blood pressure and glucose levels. Unfortunately, less than one third of the hypertensive diabetics meet their blood pressure targets and less than half of them their HbA1c target.

\section{Pharmacotherapy}

During recent years new medications and fixed dose combinations have been developed for the treatment of T2D and hypertension. For achieving glycaemic control, a number of oral therapeutic options are available, using different approaches. There are agents that increase insulin secretion, such that improve insulin action and also substances delaying carbohydrate absorption. Unfortunately, most of them - with the exception of metformin - are associated with weight gain. Hypoglycaemia and in some cases gastrointestinal side effects and oedema are other possible disadvantages. Another issue is the failure to achieve adequate control of postprandial blood glucose and also long term glycaemic control [13]. In recent years, incretin-based treatments like DPP-4 inhibitors (Vildagliptin, Sitagliptin, Saxagliptin, Linagliptin) have been shown to be a potent strategy and are increasingly used in fixed dose combinations (e.g. with metformin) [14-18]. As these drugs display different properties, e.g. pharmacokinetics, they influence the glycaemic profile in T2D - patients in different ways. This may be measure by the Mean Amplitude of Glycaemic Excursions (MAGE) and Rizzo confirmed that MAGE reduction was associated with reduction of oxidative stress and markers of systemic inflammation in T2D patients [19]. Since glucose variations over time, linked to circadian fluctuations of glucose levels, are associated with an activation of oxidative stress, the main mechanisms that lead to chronic diabetic complications [20], these data suggest that any therapy should aim not only to reduce HbA1c but also to flatten acute glucose fluctuations over time to positively influence the outcome of T2D patients.

To achieve adequate blood pressure control, an even larger number of therapy options exist. As diabetic patients frequently also have impaired renal function associated with microalbuminuria, substances affecting the renin-angiotensin system, which are known to display renal benefits independent of blood pressure reduction, appear to be particularly beneficial [21-23]. ACEI and ARB are available in several fixed dose combinations with calcium channel blockers like amlodipine and/or diuretics like hydrochlorothiazide. Interestingly, the use of fixed dose combinations has been shown to significantly improve compliance by reducing pill burden 
[24,25], which results in higher rates of treatment target achievement and lower hospitalisation rates.

\section{Guideline compliance in daily clinical practice}

Treatment recommendations by current guidelines are mainly based on evidence from randomised controlled trials. However, these trials reflect only selected patient populations defined by detailed in- and exclusion criteria of these trials. Patients in daily practice are usually older and suffer from more co-morbidities as compared to those in clinical trials $[26,27]$. Available data suggest suboptimal treatment target achievement with respect to glucose, blood pressure and lipid control [28-31]. While there are quite some data on patient characteristics, current treatments and outcome of diabetics with hypertension in clinical practice, there are much less especially with respect to the use of newly developed and approved anti-hypertensive and anti-diabetic compounds and physicians approach towards its management [32]. Additionally, it is quite unclear if and how the different combinations of anti-diabetic and anti-hypertensive medications contribute to the achievement and preservation of target blood glucose and blood pressure values in individual patients.

\section{Aim}

The purpose of the registry is to evaluate various therapy regimes of anti-diabetic (including incretin-based and exclusively non-incretin-based therapies) and antihypertensive treatments (including RAAS-inhibitors and exclusively non-RAAS-inhibitors) as well as their combinations, patient reported outcomes and treatment success in hypertensive T2D patients. It is the first prospective registry to determine treatment success based on the new individualized treatment targets of the ADA and the EASD [33].

\section{Methods/Design}

DIALOGUE is a prospective observational national multicentre registry with a follow-up of 24 months and will enrol 10,000 patients with both T2D and hypertension from up to 700 sites in Germany. Data are recorded at baseline and will be prospectively documented during follow up visits at 6,12 and 24 months.

This registry is conducted in accordance with the ethical principles that have their origin in the Declaration of Helsinki and adhere to the principles of Good Epidemiology Practice (GEP), and applicable regulatory requirements. The protocol of this registry was approved by the ethics committee of the Ruhr University Bochum, Germany. Patients that being enrolled into this registry will provide written informed consent. DIALOGUE has further been registered in the database of the Verband forschender Arzneimittelhersteller (VFA).

\section{Primary objective}

The two co-primary objectives are: 1) documentation of individual HbA1c goal achievement with respect to antidiabetic pharmacotherapy and 2) documentation of individual blood pressure goal achievement with different anti-hypertensive treatments.

\section{Secondary objective}

Secondary objectives are (1) to document major cardiovascular and cerebro-vascular events (MACCE) during 2 year follow-up; (2) to document hospitalizations during 2 year follow-up; (3) to assess the proportion of patients reaching blood glucose target values without experiencing the following adverse effects: peripheral oedema or proven hypoglycaemic events or discontinuation due to gastrointestinal events or significant weight gain $(>5 \%)$; (4) to describe patient characteristics in patients with diabetes mellitus and hypertension in clinical practice in the overall registry population; (5) to document antidiabetic and anti-hypertensive therapy and its impact on treatment target achievements in diverse subject populations, which have to be pre-specified by the scientific committee (e.g. females versus males, age </>75y, patients on insulin versus patients not on insulin, etc.); (6) to verify the applicability of and the adherence to the current guidelines for the treatment of diabetes and hypertension in clinical practice; (7) to document utilisation patterns of drugs used for the treatment of diabetes as well as hypertension in clinical practice; (8) To evaluate adverse cardio-vascular events as well as diabetesrelated micro-vascular and macro-vascular events; (9) to evaluate the glycaemic profiles of the participants with regards to differences in anti-diabetic treatment patterns; (10) to evaluate the blood pressure profiles; (11) To evaluate co-morbid disease conditions; (12) to evaluate the change in BMI over the course of the study; (13) to evaluate the proportion of patients with hypoglycaemic events over the course of the follow-up; (14) to evaluate cardio-vascular risk by using validated cardio-vascular risk scores such as the EURO Score; (15) to evaluate health status (EQ-5D); (16) to determine costs associated with the treatment and disease related complications; (17) to document treatment persistence over time, change in treatments / dosing during a follow-up of two years (optional up to 4 years of follow-up); (18) to document patient reported outcome (PRO).

\section{Selection of sites}

The registry will be performed in primary care and diabetes centres in Germany, with a planned participation of up to 700 sites. Centres will be selected from a database maintained at the Institut für Herzinfarktforschung to be representative for the ambulatory treatment of diabetes and hypertension in Germany. For this purpose, a 
representative cross-section of different types of centres including diabetologists and primary care physicians will be built. The sampling strategy will thus provide a representative dataset for the description of oral anti-diabetic treatment patterns in Germany.

\section{Selection of patients}

Inclusion criteria are as follows: 1) Age: $\geq 18$ years 2) Diagnosed type 2 diabetes mellitus and manifest hypertension (comorbidity) 3) Antidiabetic therapy presently on oral mono- or dual combination therapy (no insulin, no GLP-1 analogue) 4) The treating physician considers blood glucose lowering medication to be not adequate and/or not safe/tolerable 5) The physician adds another oral drug / switches drug treatment to achieve glycaemic control 6) Written informed consent for participation obtained from the subject.

Patients will not be eligible for inclusion if any of the following exclusion criteria apply: 1.) Current participation in any randomised controlled trial. 2) Patients not under regular supervision of the treating physician for the duration of the study 3) Use of GLP-1-analogues or insulin before enrolment 4) Patients treated with aliskiren in a dual renin angiotensin aldosterone (RAAS) blockade 5) Pregnancy 6) Diabetes secondary to malnutrition, infection or surgery 7) Maturity onset diabetes of the young 8) Known cancer.

Patients will be enrolled according to a pre-specified ratio based on their treatment, which is not predetermined by the study protocol but based on the physician's decision (Figure 1). "Incretin-based treatment" is defined as either a DPP-4 inhibitor or a GLP-1 analogue. "Non-incretin-based treatment" is defined as any of the following: metformin, sulfonylureas, acarbose, insulin, alpha-glucosidase inhibitors, and/or SGLT2-inhibitors. As the clinical profile of vildagliptin appears to differ from that of other DPP-4 inhibitors, the "incretin-based treatment" group will be split into those with or without vildagliptin.

\section{Recruitment plan}

Patient enrolment has started in July 2012. It is estimated that the first visit of the last patient enrolled will take place in early 2013, the last visit of the last patient is planned for early 2015. The Clinical Study Report will then be published in April of the following year. Figure 2 gives an overview of the timelines and the points when interim analyses to be performed.

\section{Documented variables}

Table 1 gives an overview of the variables to be documented. Source documentation and data accuracy will be verified by site visits in randomly selected $2 \%$ of the sites.

\section{Quality assurance}

There are three strategies for data quality checks: validations that occur at the time of data entry (i.e., "front-end"), a second, more sophisticated quality control program that runs as a prelude to the creation of the analysis data set and on-site data monitoring.

Front-end data checks are advantageous because mistakes are caught and corrected at the time of entry - a system that is efficient for data collectors. Certain data elements can be required, while other variables may allow for missing values. Additionally, parameters will be defined to allow entry of only those records that meet inclusion criteria.

Prior to the creation of the analytic dataset, more extensive quality control processes are performed. These checks, programmed in SAS, include parent-child edits, consistency edits, and data transformations that will facilitate analyses.

Source documentation and data accuracy will be verified by site visits in randomly selected $2 \%$ of the sites.

\section{Statistical methods}

All variables collected in the eCRF as well as the data obtained from the quality of life assessments and all

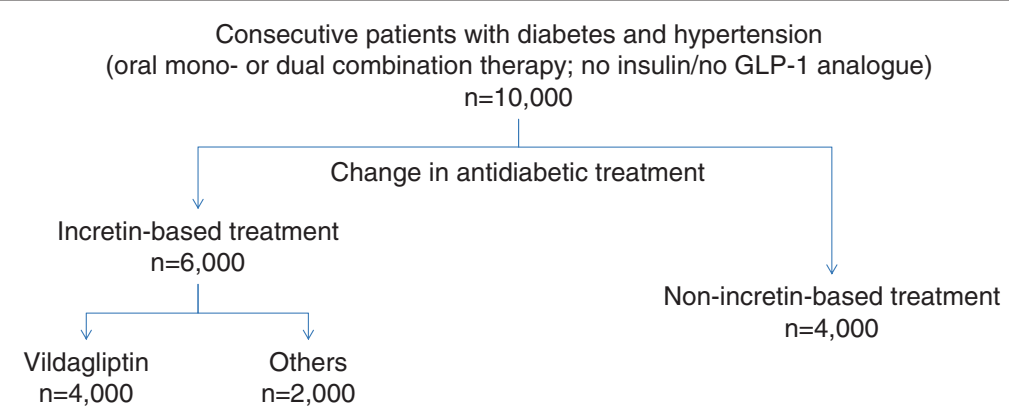

Figure 1 Sample size and segmentation into strata of different antidiabetic-treatments. Legend: Patients are eligible for inclusion if treated with oral mono or dual combination therapy and are distributed into the following groups: Incretin-based treatments include DPP-4 inhibitors and GLP-1 analogues. Non-incretin-based therapies include metformin, sulfonylureas, acarbose, insulin, alpha-glucosidase inhibitors, and SGLT2inhibitors. 


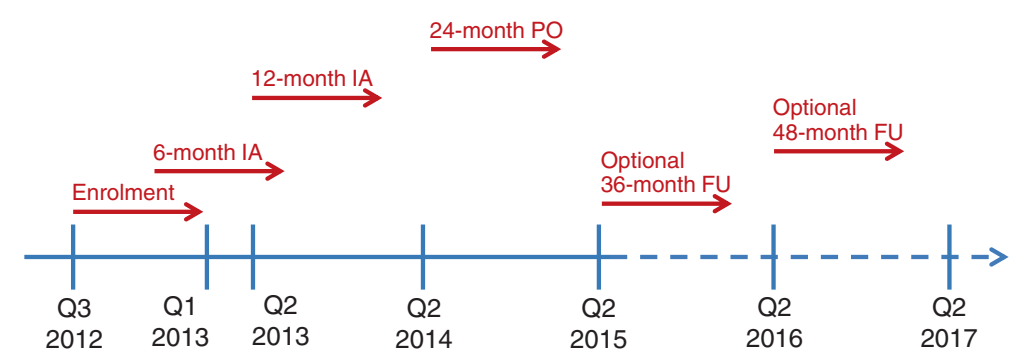

Figure 2 Estimated enrolment and planned interim-analyses as well as follow-up periods. Legend: IA, Interim Analysis, PO, Primary Objective; FU, Follow-up.

derived parameters will be used in the statistical analysis. Binary, categorical, and ordinal parameters will be summarised by means of absolute and percentage numbers within the various categories (including 'missing data' as valid category at baseline). Numerical data will be summarised by means of standard statistics (i.e. number of available data, number of missing data, mean, standard deviation, minimum, median, maximum, lower and upper quartile). In addition, adequate graphs (e.g. bar charts, box-whisker plots) may be presented to summarise the results for some parameters. Time-to-event variables will be analysed via a Cox proportional hazard regression model presenting hazard ratios and the corresponding 95\% confidence intervals. In addition Kaplan-Meier curves will be presented for these variables. Two-sided 95\%-CI will be presented for important parameters, but should be interpreted in an exploratory descriptive way. Further multivariable analyses will be performed according to the statistical analysis plan (SAP). Formal statistical tests will not be performed within the statistical analysis. A report including descriptive statistics of all documented parameters will be generated for the overall patient population. Depending on the variable(s) of interest, additional selection criteria for patients (e.g. subgroup analyses) considered in specific analyses may be used, if considered useful during the statistical analysis. Details on the selection criteria used will be given in the SAP and in the statistical section of the report. The statistical analysis will be performed using SAS (release 9.2 or higher; Cary, NC, USA).

\section{Discussion}

There are a number of epidemiological studies, which documented (among other aspects) the treatment of type- 2 diabetes in Germany. Table 2 gives an overview of those studies closely related to the objectives of DIALOGUE. These include DUTY [34], DIG [35-37] and DiaRegis [2,38-40]. DUTY (2001-2003) was among the first [34] to prospectively document the effect of a tailored intervention on blood glucose and CV risk factor target achievement. They demonstrated that too many patients suffering from diabetes mellitus do not receive consistent therapy for cardiovascular risk factors according to guidelines and therefore the required target values were

Table 1 Overview of documented parameters

\begin{tabular}{|c|c|c|c|c|}
\hline Visit & Baseline & FU 6 mo & FU $12 \mathrm{mo}$ & FU 24 mo \\
\hline Sociodemographics $^{1}$ & $x$ & & & \\
\hline Physical examination ${ }^{2}$ & $x$ & & & \\
\hline Cardiovascular concomitant diseases ${ }^{3}$ & $x$ & $x$ & $x$ & $x$ \\
\hline Diabetes associated diseases ${ }^{4}$ & $x$ & $x$ & $x$ & $x$ \\
\hline Available laboratory values ${ }^{5}$ & $x$ & $x$ & $x$ & $x$ \\
\hline Antidiabetic medication ${ }^{6}$ & $x$ & $x$ & $x$ & $x$ \\
\hline Additional current medication & $x$ & $x$ & $x$ & $x$ \\
\hline Hypoglycaemic events ${ }^{8}$ & $x$ & $x$ & $x$ & $x$ \\
\hline QoL (EuroQoL-5D) & $x$ & $x$ & $x$ & $x$ \\
\hline Patient reported outcomes (PRO) & $x$ & $x$ & $x$ & $x$ \\
\hline
\end{tabular}

Legend: 1) age, gender, insurance status, DMP participation, education, employment status, care level 2) weight, height, waist circumference, smoking, alcohol consumption, physical activity 3) coronary heart disease, previous myocardial infarction, previous PCI, previous CABG, previous stroke, heart failure (NYHA) peripheral artery occlusive disease 4) dyslipidemia, amputation, autonomous neuropathy, non- proliferative/proliferative retinopathy, diabetic macular edema, blindness, dialysis other 5) less than six weeks old: lipid values (fasting total cholesterol, fasting LDL, fasting HDL, triglycerides), fasting and post-prandial blood glucose, HbA1c, renal values (serum creatinine, microalbustix albumin, microalbustix creatinine), liver parameters. 6) metformin, sufonylureas, glucosidase inhibitors, glinides, glitazones, DPP-4 inhibitors) 7) ACE inhibitors, ARBs, renin inhibitors, beta blockers, calcium channel blockers, diuretics, other) 8 ) 12 months before baseline visit and since baseline or last FU, respectively. 
Table 2 Comparison of DIALOGUE with other existing registries

\begin{tabular}{|c|c|c|c|c|}
\hline & DIALOGUE & DiaRegis & DIG & DUTY \\
\hline Reference & & {$[2,38-40]$} & {$[35-37]$} & {$[34]$} \\
\hline No. of physicians & Up to 700 & 313 & 238 & n.a. \\
\hline No. of patients & Up to 10,000 & 3,810 & 4,020 & 59,035 \\
\hline Recruitment & Starting 06/2012 & 06/2009-03/2010 & $2002-2004$ & $2001-2003$ \\
\hline Follow-up & 2-4 years & 2 years & 4 years & 9 months \\
\hline Design & Prospective cohort & Prospective cohort & $\begin{array}{l}\text { Prospective cohort } \\
\text { study }\end{array}$ & Prospective cohort study \\
\hline $\begin{array}{l}\text { Monitoring for } \\
\text { data verification }\end{array}$ & Yes (2\%) & Yes (10\%) & None & None \\
\hline Proportion T2D & $100 \%$ & $100 \%$ & $100 \%$ & $100 \%$ \\
\hline Patients & $\begin{array}{l}\text { Co-morbid disease of diabetes and } \\
\text { hypertension }\end{array}$ & $\begin{array}{l}\text { Patients on oral mono- or dual } \\
\text { antidiabetic combination therapy }\end{array}$ & $\begin{array}{l}\text { Type-2 Diabetes } \\
\text { mellitus }\end{array}$ & $\begin{array}{l}\text { Type-1 or type-2 diabetes } \\
\text { mellitus }\end{array}$ \\
\hline $\begin{array}{l}\text { Median age } \\
\text { (years) }\end{array}$ & n.a. & 65.9 & Mean $61.8 \pm 8.1$ & $64.4 \pm 11.7$ \\
\hline Female (\%) & n.a. & 46.7 & 46.8 & 50.9 \\
\hline BMI (median) & n.a. & 30.0 & Mean $30.7 \pm 5.2$ & Mean $28.7 \pm 4.8$ \\
\hline Focus & $\begin{array}{l}\text { Target achievement with respect } \\
\text { to } \mathrm{HbA} 1 \mathrm{c} \text { and blood pressure }\end{array}$ & $\begin{array}{l}\text { Hypoglycaemia incidence with } \\
\text { antidiabetic drug use }\end{array}$ & $\begin{array}{l}\text { Application of } \\
\text { guidelines in clinical } \\
\text { practice }\end{array}$ & $\begin{array}{l}\text { Effect of tailored intervention } \\
\text { on target achievement }\end{array}$ \\
\hline
\end{tabular}

Legend. n.a., not available.

rarely reached. DIG [35-37] started one year later (2002) and had a four year follow-up and meant to document guideline-oriented treatment across Germany. A major secondary focus was to investigate the metabolic syndrome in Germany. DiaRegis [2,38-40] is a prospective registry focussing on the role of hypoglycaemia on subsequent vascular events. Throughout the follow-up a steady increase in the incidence of vascular events was documented, suggesting an association between hyperglycaemia and vascular events [41].

Most of these studies however either had a detailed look on the prevalence of type- 2 diabetes in primary care practice (HYDRA and DETECT), the co-morbidity burden (DETECT), the costs (CODE-2, CoDiM and ROSSO), or on self-monitoring of blood glucose (ROSSO). Some were also retrospective in design (CODE-2, CoDiM and ROSSO). Similar to DIALOGUE the effect of a tailored intervention on target achievement was investigated in DUTY and DIG. The added value of DIALOGUE is, that it will enrol almost 10,000 patients exclusively with the co-morbid disease constellation diabetes and hypertension and the effect of two matching treatment approaches on target goal achievement as to the more recent guidelines which suggest more individualized treatment targets $[33,42,43]$ based on increasing calls for a move toward more patient-centred care $[44,45]$.

\section{Conclusions}

DIALOGUE is the first registry that focuses at the evaluation of T2D patients who also suffer from hypertension, addressing both achievement of glycaemic and antihypertensive goals considering the individualized treatment targets as recommended by recent guidelines. As the combination of both diseases is a major factor in the development of vascular complications, data obtained will help to explain the gap between the inadequate glycaemic and blood pressure control in the "real world setting" despite the demonstrated efficacy and safety of anti-diabetic and anti-hypertensive drugs in clinical trials.

\section{Abbreviations}

MACCE: Major Cardiovascular and Cerebrovascular Events; MAGE: Mean Amplitude of Glycaemic Excursions; T2D: Type-2 Diabetes Mellitus; UKPDS: United Kingdom Prospective Diabetes Study; ACEl: ACE-inhibitor; ARB: Antiotensin Receptor Blocker; RAAS: Renin Angiotensin Aldosterone System.

\section{Competing interests}

Anselm K Gitt (AKG), Roland E. Schmieder (RES), Peter Bramlage (PB), and Diethelm Tschöpe (DT) have received research support and honoraria for lectures from a number of pharmaceutical companies producing antidiabetic drugs including Novartis the sponsor of this study. Eve Duetting (ED) is employee of the sponsor. Steffen Schneider (StS) has no potential conflict of interest to disclose.

\section{Authors' contributions}

$A K G, E D, R E S$ and DT designed the registry. StS is responsible for the analysis of data. PB drafted the manuscript based on the protocol and all other authors revised the article for important intellectual content. All authors have finally approved the version to be published.

\section{Authors' information}

DIALOGUE study group: Sibel Avsar (Ludwigshafen), Peter Bramlage (Mahlow), Eva Duetting (Nürnberg), Anselm K. Gitt (Ludwigshafen), Alexander Neumer (Ludwigshafen), Taoufik Ouarrak (Ludwigshafen), Roland E. Schmieder (Erlangen), Steffen Schneider (Ludwigshafen), Diethelm Tschöpe (Bad Oeynhausen). 


\section{Acknowledgements}

Novartis Pharma GmbH, Nürnberg, Germany funded this registry.

\section{Author details}

${ }^{1}$ Stiftung Institut für Herzinfarktforschung Ludwigshafen, Bremser Strasse 79, 67063, Ludwigshafen, Germany. ${ }^{2}$ Herzzentrum Ludwigshafen, Med. Klinik B, Ludwigshafen, Germany. ${ }^{3}$ Universitätsklinikum Erlangen, Med. Klinik 4, Schwerpunkt Nephrologie / Hypertensiologie, Erlangen, Germany. ${ }^{4}$ Novartis Pharma GmbH, Nürnberg, Germany. ${ }^{5}$ Institut für Pharmakologie und präventive Medizin, Mahlow, Germany. 'Stiftung ,Der herzkranke Diabetiker" in der Deutschen Diabetes-Stiftung, Georgstrasse 11, 32545, Bad Oeynhausen, Germany. ${ }^{7}$ Herz- und Diabeteszentrum Nordrhein-Westfalen in Bad Oeynhausen, Universitätsklinik der Ruhr Universität Bochum, Germany.

Received: 31 October 2012 Accepted: 27 November 2012 Published: 5 December 2012

\section{References}

1. Wild S, Roglic G, Green A, Sicree R, King H: Global prevalence of diabetes: estimates for the year 2000 and projections for 2030. Diabetes Care 2004, 27(5):1047-1053.

2. Bramlage P, Binz C, Gitt AK, Krekler M, Plate T, Deeg E, Tschope D: Diabetes treatment patterns and goal achievement in primary diabetes care (DiaRegis) - study protocol and patient characteristics at baseline. Cardiovasc Diabetol 2010, 9:53.

3. Haffner SM, Lehto S, Ronnemaa T, Pyorala K, Laakso M: Mortality from coronary heart disease in subjects with type 2 diabetes and in nondiabetic subjects with and without prior myocardial infarction. N Engl J Med 1998, 339(4):229-234.

4. Zomer E, Liew D, Owen A, Magliano DJ, Ademi Z, Reid CM: Cardiovascular risk prediction in a population with the metabolic syndrome: Framingham vs. UKPDS algorithms. Eur J Prev Cardio/ 2012, doi:10.1177/ 2047487312449307 (published ahead of print).

5. The Diabetes Control and Complications Trial Research Group: The effect of intensive treatment of diabetes on the development and progression of long-term complications in insulin-dependent diabetes mellitus. N Engl J Med 1993, 329(14):977-986.

6. UKPDS: Intensive blood-glucose control with sulphonylureas or insulin compared with conventional treatment and risk of complications in patients with type 2 diabetes (UKPDS 33). Lancet 1998, 352(9131):837-853.

7. Ray KK, Seshasai SR, Wijesuriya S, Sivakumaran R, Nethercott S, Preiss D, Erqou S, Sattar N: Effect of intensive control of glucose on cardiovascular outcomes and death in patients with diabetes mellitus: a meta-analysis of randomised controlled trials. Lancet 2009, 373(9677):1765-1772.

8. Adler Al, Stratton IM, Neil HA, Yudkin JS, Matthews DR, Cull CA, Wright AD, Turner RC, Holman RR: Association of systolic blood pressure with macrovascular and microvascular complications of type 2 diabetes (UKPDS 36): prospective observational study. BMJ 2000, 321(7258):412-419.

9. Gaede P, Valentine WJ, Palmer AJ, Tucker DM, Lammert M, Parving $\mathrm{HH}_{\text {, }}$ Pedersen O: Cost-effectiveness of intensified versus conventional multifactorial intervention in type 2 diabetes: results and projections from the Steno-2 study. Diabetes Care 2008, 31(8):1510-1515.

10. Gaede P, Vedel P, Larsen N, Jensen GV, Parving HH, Pedersen O: Multifactorial intervention and cardiovascular disease in patients with type 2 diabetes. N Engl J Med 2003, 348(5):383-393.

11. Mancia G, Laurent S, Agabiti-Rosei E, Ambrosioni E, Burnier M, Caulfield MJ, Cifkova R, Clement D, Coca A, Dominiczak A, et al: Reappraisal of European guidelines on hypertension management: a European Society of Hypertension Task Force document. Blood Press 2009, 18(6):308-347.

12. Matthaei $S$, Bierwirth $R$, Fritsche $A$, Gallwitz $B$, Häring $H-U$, Joost $H-G$, Kellerer M, Kloos C, Kunt T, Nauck M, et al: [Treatment of type-2 diabetes mellitus]. Diabetologie 2011, 6(Suppl. 2):S131-S136.

13. Cefalu WT: Evolving treatment strategies for the management of type 2 diabetes. Am J Med Sci 2012, 343(1):21-26.

14. Ferrannini E, Fonseca V, Zinman B, Matthews D, Ahren B, Byiers S, Shao Q, Dejager S: Fifty-two-week efficacy and safety of vildagliptin vs. glimepiride in patients with type 2 diabetes mellitus inadequately controlled on metformin monotherapy. Diabetes Obes Metab 2009, 11(2):157-166.

15. Matthews DR, Dejager S, Ahren B, Fonseca V, Ferrannini E, Couturier A, Foley JE, Zinman B: Vildagliptin add-on to metformin produces similar efficacy and reduced hypoglycaemic risk compared with glimepiride, with no weight gain: results from a 2-year study. Diabetes Obes Metab 2010, 12(9):780-789.

16. Filozof C, Gautier JF: A comparison of efficacy and safety of vildagliptin and gliclazide in combination with metformin in patients with Type 2 diabetes inadequately controlled with metformin alone: a 52-week, randomized study. Diabet Med 2010, 27(3):318-326.

17. Goke B, Gallwitz B, Eriksson J, Hellqvist A, Gause-Nilsson I: Saxagliptin is non-inferior to glipizide in patients with type 2 diabetes mellitus inadequately controlled on metformin alone: a 52-week randomised controlled trial. Int J Clin Pract 2010, 64(12):1619-1631.

18. Forst T, Uhlig-Laske B, Ring A, Graefe-Mody U, Friedrich C, Herbach K, Woerle HJ, Dugi KA: Linagliptin (BI 1356), a potent and selective DPP-4 inhibitor, is safe and efficacious in combination with metformin in patients with inadequately controlled Type 2 diabetes. Diabet Med 2010, 27(12):1409-1419.

19. Rizzo MR, Barbieri M, Marfella R, Paolisso G: Reduction of Oxidative Stress and Inflammation by Blunting Daily Acute Glucose Fluctuations in Patients With Type 2 Diabetes: Role of dipeptidyl peptidase-IV inhibition. Diabetes Care 2012, 35(10):2076-2082.

20. Monnier L, Mas E, Ginet C, Michel F, Villon L, Cristol JP, Colette C: Activation of oxidative stress by acute glucose fluctuations compared with sustained chronic hyperglycemia in patients with type 2 diabetes. JAMA 2006, 295(14):1681-1687.

21. Haller H, Ito S, Izzo JL Jr, Januszewicz A, Katayama S, Menne J, Mimran A, Rabelink TJ, Ritz E, Ruilope LM, et al: Olmesartan for the delay or prevention of microalbuminuria in type 2 diabetes. N Engl J Med 2011, 364(10):907-917.

22. Shishido T, Konta T, Nishiyama S, Miyashita T, Miyamoto T, Takasaki S, Nitobe J, Watanabe T, Takeishi Y, Kubota I: Suppressive effects of valsartan on microalbuminuria and CRP in patients with metabolic syndrome (Val-Mets). Clin Exp Hypertens 2011, 33(2):117-123.

23. Schmieder RE, Hilgers KF, Schlaich MP, Schmidt BM: Renin-angiotensin system and cardiovascular risk. Lancet 2007, 369(9568):1208-1219.

24. Bangalore S, Kamalakkannan G, Parkar S, Messerli FH: Fixed-dose combinations improve medication compliance: a meta-analysis. Am J Med 2007, 120(8):713-719.

25. Bramlage $P$, Hasford $\mathrm{J}$ : Blood pressure reduction, persistence and costs in the evaluation of antihypertensive drug treatment-a review. Cardiovasc Diabetol 2009, 8:18.

26. Bramlage $P$, Wolf WP, Stuhr T, Fronk EM, Erdlenbruch W, Ketelhut R, Schmieder RE: Effectiveness and tolerability of a fixed-dose combination of olmesartan and amlodipine in clinical practice. Vasc Health Risk Manag 2010, 6:803-811

27. Stolar MW, Hoogwerf BJ, Gorshow SM, Boyle PJ, Wales DO: Managing type 2 diabetes: going beyond glycemic control. J Manag Care Pharm 2008, 14(5 Suppl B):s2-s19.

28. Braga MF, Casanova A, Teoh H, Gerstein HC, Fitchett DH, Honos G, McFarlane PA, Ur E, Yale JF, Langer A, et al: Poor achievement of guidelines-recommended targets in type 2 diabetes: findings from a contemporary prospective cohort study. Int J Clin Pract 2012, 66(5):457-464.

29. Ruckert IM, Schunk M, Holle R, Schipf S, Volzke H, Kluttig A, Greiser KH, Berger K, Muller G, Ellert U, et al: Blood pressure and lipid management fall far short in persons with type 2 diabetes: results from the DIAB-CORE Consortium including six German population-based studies. Cardiovasc Diabetol 2012, 11:50.

30. Chittleborough CR, Baldock KL, Phillips PJ, Taylor AW: Achievement of management targets associated with incident and long-term diagnosed diabetes among a representative population sample. Diabetes Res Clin Pract 2010, 88(3):322-327.

31. Kuznik A, Mardekian J: Trends in utilization of lipid- and blood pressure-lowering agents and goal attainment among the U.S. diabetic population, 1999-2008. Cardiovasc Diabetol 2011, 10:31.

32. Owen AJ, Retegan C, Rockell M, Jennings G, Reid CM: Inertia or inaction? Blood pressure management and cardiovascular risk in diabetes. Clin Exp Pharmacol Physiol 2009, 36(7):643-647.

33. Inzucchi SE, Bergenstal RM, Buse JB, Diamant M, Ferrannini E, Nauck M, Peters AL, Tsapas A, Wender R, Matthews DR: Management of hyperglycemia in type 2 diabetes: a patient-centered approach: position statement of the American Diabetes Association (ADA) and the 
European Association for the Study of Diabetes (EASD). Diabetes Care 2012, 35(6):1364-1379.

34. Krone W, Bohm M, Wöhrmann APA, Bestehorn K: Erhebung und Verbesserung der Behandlungssituation von Patienten mit Diabetes mellitus. Das DUTY-Register. Bundesgesundheitsblatt Gesundheitsforschung Gesundheitsschutz 2004, 47(6):540-546.

35. Ott P, Köhler C, Hanefeld M: Baseline data of the Diabetes in Germany study (DIG). Prospective 4-year study on the epidemiology of type-2 diabetes in Germany. Diabetologe 2006, 2(Suppl. 1):S44-S48.

36. Hanefeld M, Koehler C, Gallo S, Benke I, Ott P: Impact of the individual components of the metabolic syndrome and their different combinations on the prevalence of atherosclerotic vascular disease in type 2 diabetes: the Diabetes in Germany (DIG) study. Cardiovasc Diabetol 2007, 6:13.

37. Koehler C, Ott P, Benke I, Hanefeld M, Group DIGS: Comparison of the prevalence of the metabolic syndrome by WHO, AHA/NHLBI, and IDF definitions in a German population with type 2 diabetes: the Diabetes in Germany (DIG) Study. Horm Metab Res 2007, 39(9):632-635.

38. Gitt AK, Bramlage P, Binz C, Krekler M, Deeg E, Tschope D: [Comorbidity, hypoglycaemia and appropriate selection of antidiabetic pharmacotherapy in diabetic patients with heart failure in clinical practice in Germany. Results of the DiaRegis registry]. Herz 2012, 37(3):294-300.

39. Halle M, Gitt AK, Hanefeld M, Kellerer M, Marx N, Meier Jj, Schumm-Draeger PM, Bramlage P, Tschoepe D: [Diabetes and heart failure: a practically oriented critical appraisal]. Dtsch Med Wochenschr 2012, 137(9):437-441.

40. Gitt AK, Bramlage P, Binz C, Krekler M, Plate T, Deeg E, Tschope D: Hypoglycaemia is more frequent in type 2 diabetic patients with co-morbid vascular disease: an analysis of the DiaRegis registry. Eur J Prev Cardiol 2012, 19(4):765-772.

41. Tschope D, Bramlage P, Binz C, Krekler M, Plate T, Deeg E, Gitt AK: Antidiabetic pharmacotherapy and anamnestic hypoglycemia in a large cohort of type 2 diabetic patients-an analysis of the DiaRegis registry. Cardiovasc Diabetol 2011, 10:66.

42. American Diabetes A: Standards of medical care in diabetes-2012. Diabetes Care 2012, 35(Suppl 1):S11-S63.

43. Mancia G, Laurent S, Agabiti-Rosei E, Ambrosioni E, Burnier M, Caulfield MJ, Cifkova R, Clement D, Coca A, Dominiczak A, et al: Reappraisal of European guidelines on hypertension management: a European Society of Hypertension Task Force document. J Hypertens 2009, 27(11):2121-2158.

44. Murad MH, Shah ND, Van Houten HK, Ziegenfuss JY, Deming JR, Beebe TJ, Smith SA, Guyatt GH, Montori VM: Individuals with diabetes preferred that future trials use patient-important outcomes and provide pragmatic inferences. J Clin Epidemiol 2011, 64(7):743-748.

45. Glasgow RE, Peeples M, Skovlund SE: Where is the patient in diabetes performance measures? The case for including patient-centered and self-management measures. Diabetes Care 2008, 31(5):1046-1050.

doi:10.1186/1475-2840-11-148

Cite this article as: Gitt et al: Achievement of recommended glucose and blood pressure targets in patients with type 2 diabetes and hypertension in clinical practice - study rationale and protocol of DIALOGUE. Cardiovascular Diabetology 2012 11:148.

\section{Submit your next manuscript to BioMed Central and take full advantage of:}

- Convenient online submission

- Thorough peer review

- No space constraints or color figure charges

- Immediate publication on acceptance

- Inclusion in PubMed, CAS, Scopus and Google Scholar

- Research which is freely available for redistribution

Submit your manuscript at www.biomedcentral.com/submit
C Biomed Central 